\title{
The Analysis of Performance Measures of Generalized Trapezoidal Fuzzy Queuing Model with an Unreliable Server
}

\author{
V.R. Bindu Kumari ${ }^{1,}$ Dr. R. Govindarajan ${ }^{2}$ \\ ${ }^{I}$ Assistant Professor, Department of Mathematics, St. Thomas College of Arts \& Science, Koyambedu, \\ Chennai-600 107, Tamilnadu, India. \\ ${ }^{2}$ Rtd. Head \& Professor, PG \& Research Department of Mathematics, D.G. Vaishnav College, Chennai-600 \\ 106, Tamilnadu, India.
}

\begin{abstract}
The purpose of this research paper was to propose a method which can be utilized to determine the different types of performance measures on the basis of the crisp values for the fuzzy queuing model which has an unreliable server and where the rate of arrival, the rate of service, the rate of breakdown and the rate of repair are all expressed as the fuzzy numbers. In this case the inter arrival time, the time of service, the rates of breakdown and the rates of repair are all triangular functions and are also expressed as the Trapezoidal fuzzy numbers. The main intent is to transform the fuzzy inter arrival time, the time of service, the rates of breakdown and the rates of repair into the crisp values by using the Ranking function method. Then the crisp values are applied in the classical formulas for the performance measure. In the fuzzy environment the ranking fuzzy numbers are very helpful in making the decisions. The ranking function method is one of the most reliable method, is simpler to apply in comparison to other methods and can be utilized to solve the different types of queuing problems. In this research paper a numerical example is also provided for both the triangular and the trapezoidal fuzzy number so that a practical insight into the problem can be provided.
\end{abstract}

Key words: Fuzzy sets, Fuzzy queues, Fuzzy ranking, Membership Functions, Unreliable server

\section{INTRODUCTION}

It is not always possible to keep the server in operational mode at all times in most of the queuing models which can lead to the interruption in the services. The main reason behind the interruption of the services is that the server breaks down. Also during the weekends and the holidays there can be the schedules service breakdown of the server. The first ordinary M/G/I queuing system which has interrupted services and priorities was first proposed by Gaver [1]. Then Sengupta [2] extended the system proposed by Gaver [1] to the GI/G/I case. The behavior of the of the unreliable server and the impact of the unreliable server on the server breakdowns and repairs of the M/G/I queuing models was first established by the $\mathrm{Li}$ [3] and Wang [4]. They also analyzed the controllable M/HK/I queuing system with the unreliable server. The complex two unit system whereby the facility for repair is lead to the random breakdown is analyzed by Gurukajan and Srinivasan [5]. With the help of the extension principle conceptualized by Zadeh [6], the fuzzy queue with an unreliable server is transformed in to a family of crisp queues with an unreliable server. Taking into consideration the notational convenience, the researcher in this research paper has denoted the model by FM/FM (FM, FM)/I. In the given model the first FM represents the fuzzified exponential arrival, the second FM represents the service, the third FM depicts the breakdown and the final FM represents the rate of repair. On the contrary in case of the $\mathrm{M} / \mathrm{M}$ $(\mathrm{M}, \mathrm{M}) / \mathrm{I}$ model first to the fourth represents the exponential inter-arrival (Poisson), service, breakdown and the rates of repair respectively.

Jain [7] first proposed the method of ranking. For the purpose of ordering of the fuzzy quantities in [0, 1], Yager [8] conceptualized the four indices. A method for the ranking of the fuzzy numbers is given by the Kaufman and Gupta [9]. A subjective method for the ranking of the fuzzy numbers is given by the Campos and Gonzalez [10]. Further Liou and Wang [11] proposed a ranking method which was based on the integral value index. Another ranking method was presented by Cheng [12] which was based on the distance method. Interestingly, the overall possibility distributions of the fuzzy numbers were considered in their evaluation by Kwang and Lee [13], on the basis of which they proposed a ranking method. A ranking method was proposed by Modarres and Nezhad [14] which was dependent upon the preference function that evaluates the fuzzy numbers on a point by point basis and at every point the most preferred number is determined. A centroid index method for the ranking of the fuzzy numbers was presented by the Deng and Liu [15]. The concept of centriod was utilized by Liang et al. [16] and Wang and Lee [17], in order to develop the ranking index. The method for the ranking of the generalized trapezoidal fuzzy numbers was presented by Chen and Chen [18]. An interesting 
method for the ranking of the trapezoidal fuzzy numbers was introduced by Abbasbandy and Hajjari [19], on the basis of the left and the right spreads at certain $\boldsymbol{\alpha}$-levels of trapezoidal fuzzy numbers. Different other researchers have also analyzed the ranking methods.

In the main intent behind presenting this particular research paper is to develop a method which will enable to provide performance measures in terms of the crisp numbers for the fuzzy queuing model utilizing an unreliable server. This method will be based on four fuzzy variables which will be fuzzified exponential arrival, rate of service, rate of breakdown and rate of repair. In order to reach the crisp values the ranking function method has been utilized.

\section{Definition}

A membership function mapping the elements of the domain space or the universe of discourse $\mathrm{Z}$ to the unit interval $[0,1]$ is utilized for the determination of the fuzzy set.

i.e $\tilde{\mathrm{A}}=\left\{\left(\mathrm{z}, \mu_{\overline{\mathrm{A}}}(\mathrm{z})\right) ; \mathrm{z} \in \mathrm{Z}\right\}$.

In the above equation $\mu_{\bar{A}}: Z \rightarrow[0,1]$ is a mapping which is known as the degree of the membership function of the fuzzy set $\tilde{A}$ and $\mu_{A^{-}}(z)$ is said to the value of membership of $z \in Z$ in the fuzzy set $\tilde{A}$ The membership grades are normally represented using the real numbers between the range $[0,1]$.

Also, in the universe of discourse $\mathrm{Z}$ a fuzzy set of $\tilde{\mathrm{A}}$ is known as the normal fuzzy set is there is atleast one $\mathrm{z} \in \mathrm{Z}$ exist so that $\mu_{\overline{\mathrm{A}}}(\mathrm{z})=1$.

i) The fuzzy set can be explained as a membership function mapping element for a space in a domain or in a universe of discourse $\mathrm{X}$ for the unit of interval $[0,1]$. For example $\left.A=\left\{x, \mu_{A}(x)\right) ; x \in X\right\}$, in that case $\mu_{A}: \mathrm{X} \quad[0,1]$, can be said to be mapping which can be described as the degree of membership function of the fuzzy set A. Here $\mu_{A}(\mathrm{x})$ can be said to the membership value that is related to $\mathrm{x} \in \mathrm{X}$ of the fuzzy set A. These grades of membership are generally represented by real numbers within the range of $[0$, 1].

ii) The normal fuzzy can be explained as the fuzzy set $A$ that belongs to the universe of discourse $X$ and points out that there is atleast one $\mathrm{x} \in \mathrm{X}$ in such a manner that $\mu_{A}(\mathrm{x})=1$.

iii) The fuzzy set A can be said to be convex fuzzy set only if for any $x_{1}, x_{2} \in X$, the inequality $\mu_{A}$ $\left\{\lambda \mathrm{x}_{1}+(1-\lambda) \mathrm{x}_{2}\right\} \geq \min \left\{\mu_{A}\left(\mathrm{x}_{1}\right), \mu_{A}\left(\mathrm{x}_{2}\right)\right\} .0 \leq \lambda \leq 1$, has to be satisfied by the membership function $A$.

iv) A trapezoidal fuzzy number can be represented as A (a,b,c,d;1) which has the membership function $\mu(\mathrm{x})$ is basically expressed as :

$$
\begin{aligned}
\mu(\mathrm{x})= & (\mathrm{x}-\mathrm{a}) /(\mathrm{b}-\mathrm{a}), \mathrm{a} \leq \mathrm{x} \leq \mathrm{b} \\
& 1, \mathrm{~b} \leq \mathrm{x} \leq \mathrm{c} \\
& (\mathrm{d}-\mathrm{x}) /(\mathrm{d}-\mathrm{c}), \mathrm{c} \leq \mathrm{x} \leq \mathrm{d}
\end{aligned}
$$

\section{0 , otherwise}

v) The $\alpha$-cut of a fuzzy number $\mathrm{A}(\mathrm{x})$ is generally defined as:

$\mathrm{A}(\alpha)=[\mathrm{x}: \mu(\mathrm{x}) \geq \alpha, \alpha \in[0,1]\}$

It is possible to add two trapezoidal fuzzy numbers in the following manner:

$\left(\mathrm{a}_{1}, \mathrm{~b}_{1}, \mathrm{c}_{1}, \mathrm{~d}_{1}\right)+\left(\mathrm{a}_{2}, \mathrm{~b}_{2}, \mathrm{c}_{2}, \mathrm{~d}_{2}\right)=\left(\mathrm{a}_{1}+\mathrm{a}_{2}, \mathrm{~b}_{1}+\mathrm{b}_{2}\right.$, $\left.\mathrm{c}_{1}+\mathrm{c}_{2}, \mathrm{~d}_{1}+\mathrm{d}_{2}\right)$.

vi) Generalized fuzzy number: A generalized fuzzy number may be defined as the fuzzy set $\tilde{A}$, which can be measured on the universal set of the real numbers $\mathrm{R}$, if the membership function has the below given characteristics:

a) $\mu_{A}: \mathrm{R} \rightarrow[0, \mathrm{w}]$ is continuous;

b) $\mu_{A}(\mathrm{z})=0$ for all $\mathrm{z} \in\left(-\infty, a_{1}\right] \cup\left[a_{4}, \infty\right)$;

c) $\mu_{A}$ (z) strictly increasing on $\left[a_{1}, a_{2}\right]$ and strictly

decreasing on $\left[a_{3}, a_{4}\right]$;

d) $\mu_{A} \sim(\mathrm{z})=\mathrm{w}$, for all $\mathrm{z} \in\left[a_{2}, a_{3}\right]$, where $0<\mathrm{w} \leq 1$.

ix) Gneralized trapezoidal fuzzy numbers : The generalised trapezoidal fuzzy numbers $\tilde{A}(\mathrm{z})$ is generally expressed as $\tilde{A}\left(a_{1}, a_{2}, a_{3}, a_{4}, 1\right)$, where the mebership function is illustrated as:

$$
\mu_{\overrightarrow{\mathrm{A}}}(\mathrm{z})=\left\{\begin{array}{l}
{\left[\mathrm{w}\left(\mathrm{z}-\mathrm{a}_{1}\right) /\left(\mathrm{a}_{2}-\mathrm{a}_{1}\right)\right], a_{1} \leq z \leq a_{2}} \\
w \quad, a_{2} \leq z \leq a_{3} \\
{\left[\mathrm{w}\left(\mathrm{z}-\mathrm{a}_{4}\right) /\left(\mathrm{a}_{3}-\mathrm{a}_{4}\right)\right], a_{3} \leq z \leq a_{4}} \\
0, \text { otherwise }
\end{array}\right.
$$

\section{Fuzzy Queuing Model With An Unreliable Server}

There are two major groups in which the queuing models can be divided into. One of the group can be said to be those group which describes the actual events or real events and the other group provides an optimum view point about the what should be aspired. The descriptive models basically describes the average values and probabilities that are related to the performance measures which can be utilized to describe the system after the completion of the setting up of the patterns of arrivals and the service, the server numbers, the capacity of the system and the discipline of the queue. On the other hand the second category of models which are also termed as the queuing decision models is basically utilized to estimate the different parameters which can be used to optimize the models. In general the basic system of queuing generally consists of the queues that are organized and the units are generally dealt according to their order of arrival. In most of the queuing models the waiting discipline can be seen, but there are certain privileged classes of units which hold certain degree of priority in the work mode of the system are defined as per their efficiency and hierarchy. For example the status of the message 
that has to be transmitted in a system of telecommunication. The priority discipline model is generally followed more closely by the real queuing system than any other model for queuing that are available in the present times. In the priority model the urgent tasks are performed first than the other tasks and the main clients are given special preferences.

There have been several methods which have been proposed so as to resolve the design and the control of the queuing models in case when the coefficients of the costs and the arrival or the patterns of the service are known exactly. In certain conditions these parameters can be said to be not precise, thus in that case the unit cost of waiting overtime for every client can vary overtime. The main problem that has been found in such type of models with that of the fuzzy cost efficient is that it can be very difficult to make a proper estimate of the waiting unit cost, most importantly when the nature of the human affects the working of the system. Due to the wide range of situations the cost of service and the costs related to waiting and inactivity can be highly uncertain, which are beyond the control of the humans. In case of the conventional queuing decision models, the quality of the decision making can be undermined by the initial fuzzy information. This point out the need to study in greater depth the problems related to decision making which has the fuzzy queuing models.

Let us take into consideration a fuzzy queuing system which has an unreliable server and the there are two different types of breakdown of the system. In the type 1 of breakdown, there can be breakdown of the server where there are no customers present in the system and in the type 2 of breakdown, there is a breakdown of the server when there is at least one customer present at the system. Let us assume that the arrival of the customers takes place in a single-server facility like that of a Poisson process and have the fuzzy rate of $\lambda$. Furthur let us assume that the service times are in the form of the exponential distribution which has the fuzzy rate $\mu$, and following the Poisson process the serverr might have the breakdown which has the fuzzy rate of $\alpha$ and finnaly exponential distribution which is followed by the repair has the fuzzy rate as $\beta$. Let these functions are known approximately as the convex fuzzy sets. let us take into consideration that the memebership functions of the $\lambda, \mu, \alpha$ and $\beta$ are expressed by $\phi_{\lambda}(\mathrm{x}), \phi_{\mu}$ (y), $\phi_{\alpha}$ (s) and $\phi_{\beta}(\mathrm{t})$. Thus we get the following fuzzy sets:

$\lambda=\left\{\left(\mathrm{x}, \phi_{\lambda}(\mathrm{x})\right) \mid \mathrm{x} \in \mathrm{X}\right\}$

$\mu=\left\{\left(\mathrm{y}, \phi_{\mu}(\mathrm{y})\right) \mid \mathrm{y} \in \mathrm{Y}\right\}$

$\alpha=\left\{\left(\mathrm{s}, \phi_{\alpha}(\mathrm{s})\right) \mid \mathrm{s} \in \mathrm{S}\right\}$

$\beta=\left\{\left(\mathrm{t}, \phi_{\beta}(\mathrm{t})\right) \mid \mathrm{t} \in \mathrm{T}\right\}$

in the above equation $\mathrm{X}, \mathrm{Y}, \mathrm{S}$ and $\mathrm{T}$ can be defined as the crisp universal sets of the arrival rates, rates of service, rates of breakdown and the rates of repair respectively. Let us assume that $\mathrm{f}(\mathrm{x}, \mathrm{y}, \mathrm{s}, \mathrm{t})$ represents the characterists of the system which is of interest. As $\mathrm{x}, \mathrm{y}, \mathrm{s}, \mathrm{t}$ are denoted as the fuzzy numbers thus $\mathrm{f}(\mathrm{x}, \mathrm{y}, \mathrm{s}, \mathrm{t})$ are also fuzzy numbers. Let us take into assumption that the membership function of the expected time for which the system will remain idle in the breakdowns of type 1 and type 2, be expressed by $\mathrm{A}$ and $\mathrm{B}$.

then,

$\mathrm{A}=\mathrm{f}(\mathrm{x}, \mathrm{y}, \mathrm{s}, \mathrm{t})=\frac{t y-x(s+t)}{y(s+t)}$

and, $\mathrm{B}=\mathrm{f}(\mathrm{x}, \mathrm{y}, \mathrm{s}, \mathrm{t})=\underline{t y-x(s+t)}$

ty

In case of the steady state, it is necessary that $0<$ $\underline{t y-x(s+t)}<1$ and

$y(s+t)$

$0<\frac{t y-x(s+t)}{t y}<1$.

\section{Ranking Function Method - Algorithm}

In order to solve the problems that has been provided we have to first defuzzify the fuzzy numbers into the crisp numbers, by utilizing the fuzzy numbers Ranking function $\mathrm{R}: \mathrm{F}(\mathrm{R}) \rightarrow \mathrm{R}$ where $F(R)$ is a set of fuzzy numbers which are generally expressed on the set of real numbers, which is used for the mapping of each fuzzy numbers on the real line, where a natural order exists.

Let the convex trapezoidal fuzzy number $\tilde{A}(\mathrm{z})=\tilde{A}\left(a_{1}, a_{2}, a_{3}, a_{4}: \mathrm{w}\right)$,

$R(\tilde{A})=\frac{w\left(a_{1}+a_{2}+a_{3}+a_{4}\right)}{4}$

\section{Numerical Example}

Let us take into consideration the railway computer reservation system where the passengers are utilising a single channel reservation arrival as per the poisson process. There may be interruption in the time taken for the reservation process (due to the problems in the computer, server or the problems in the printers) according to the Poisson process. The time of recovery of the problems caused due to interruption is followed by the exponential distribution. When the interruption is over the reservation process resumes again. The railway wishes to evaluate the total number of hours the system has been in idle condition so as to analyse the single-channel reservation system. It is known that this system will follow the FM/FM(FM,FM)/1/ $\infty$ and the expected time for which the system is going to remain idle can be calculated from the procedure that has been proposed below. 


\section{Solution}

Now let us take into consideration that arrival rate, rate of service, rate of breakdown and the rates of repair are the trapezoidal fuzzy numbers which are generally expressed as :

$\lambda=[2,4,5,7 ; 1], \mu=[12,14,16,20 ; 1]$,

$\alpha=[0.05,0.1,0.2,0.5 ; 1]$,

$\beta=[2,3,5,6 ; 1]$ per hour.

As per equation we can write that, The ranking index of $\lambda$ is given by,

$\mathrm{R}(\lambda)=\mathrm{R}(2,4,5,7 ; 1)=\frac{(2+4+5+7)}{4}=4.5$

Progressing furthur on the similar basis we get,

$\mathrm{R}(\mu)=\mathrm{R}(12,14,16,20 ; 1)=$

$\frac{(12+14+16+20)}{4}=15.5$

$\mathrm{R}(\alpha)=\mathrm{R}(0.05,0.1,0.2,0.5 ; 1)=$

$\underline{(0.05+0.1+0.2+0.5)}=0.2125$

$\mathrm{R}(\beta)=\mathrm{R}(2,3,5,6 ; 1)=\frac{(2+3+5+6)}{4}=4$

As per the equations we can write that,

The expected time for which the sysytem will remain idle can be expressed as:

$$
\begin{gathered}
\text { Type 1: } \frac{t y-x(s+t)}{y(s+t)} \\
=\frac{4(15.5)-4.25(0.2125+4)}{15.5(0.2125+4)}=0.659 \\
\text { Type } 2: \frac{t y-x(s+t)}{t y} \\
=\frac{415.5)-4.5(0.2125+4)}{4(15.5)}=0.694
\end{gathered}
$$

\section{CONCLUSION}

In this particular research paper, the Fuzzy set theory has been applied on a queuing model which has an unreliable server. For the evaluation of the system performance the fuzzy queuing model with the unreliable server has put in use for the operations and the mechanisms for service. With the help of the ranking indices the fuzzy problem has been transformed into the crisp problem. As the performance measures like that of the expected time for which the system will remain idle for two types are the crisp values and thus it will be helpful for the manager to take best and the optimum decisions. Finally it is concluded that the Ranking function method is a very effective way which can be utilized to find out the problems related to the fuzzy queues with an unreliable server. The approach that has been shown in this research paper gives a practical insight for the operations and systems managers and the practitioners.

\section{REFERENCES}

[1]. W. Li, D. Shi, X. Chao, Reliability analysis of $\mathrm{M} / \mathrm{G} / \mathrm{I}$ queuing systems with server breakdowns and vacations, Journal of Applied probability 34 (1997) 546-555.

[2]. K.H. Wang, H.J. Kao, G. Chen, Optimal management of aremarkable and nonreliable server in an infinite and a finite $\mathrm{M} / \mathrm{Hk} / \mathrm{I}$ queuing systems ,Quality Technology and quantitative Management 1(2) (2004) 325-339.

[3]. Gurukajan, M. and Srinivasan, B. (1995). A complex two unit system with random breakdown of repair facility. Microelectronics and Reliability, 35(2): 299322.

[4]. L.A. Zadeh, Fuzzy sets as a basis for a theory of possibility, fuzzy sets and systems 1 (1978) 3-28.

[5]. Jain $\mathrm{R}$ (1976) Decision-making in the presence of fuzzy variables. IEEE Transactions on Systems,Fuzzy Inf. Eng. (2010) 1: 37-47 47

[6]. Yager R R (1981) A procedure for ordering fuzzy subsets of the unit interval. Information Sciences 24(2): 143

[7]. Kaufmann A, Gupta M M (1988) Fuzzy mathematical models in engineering and management science. Elsevier Science Publishers, Amsterdam, Netherlands

[8]. Campos L, Gonzalez A (1989) A subjective approach for ranking fuzzy numbers. Fuzzy Sets and Systems 29(2): 145

[9]. Liou T S, Wang M J (1992) Ranking fuzzy numbers with integral value. Fuzzy Sets and Systems 50(3): 247

[10]. Cheng C H (1998) A new approach for ranking fuzzy numbers by distance method. Fuzzy Sets and Systems 95(3): 307

[11]. Kwang H C, Lee J H (1999) A method for ranking fuzzy numbers and its application to decision making. IEEE Transaction on Fuzzy Systems 7(6): 677

[12]. Modarres M, Sadi Nezhad S (2001) Ranking fuzzy numbers by preference ratio. Fuzzy Sets and Systems 118(3): 429 